\title{
Exponential Attractors for Parabolic Equations with Dynamic Boundary Conditions
}

\author{
Zhao-hui Fan \\ Department of Mathematics, Zhengzhou University, Zhengzhou, Henan 450001, China \\ Correspondence should be addressed to Zhao-hui Fan; fanzhh02@163.com
}

Received 24 December 2012; Accepted 2 February 2013

Academic Editor: Debasish Roy

Copyright (C) 2013 Zhao-hui Fan. This is an open access article distributed under the Creative Commons Attribution License, which permits unrestricted use, distribution, and reproduction in any medium, provided the original work is properly cited.

We study exponential attractors for semilinear parabolic equations with dynamic boundary conditions in bounded domains. First, we give the existence of the exponential attractor in $L^{2}(\Omega) \times L^{2}(\Gamma)$ by proving that the corresponding semigroup satisfies the enhanced flattering property. Second, we apply asymptotic a priori estimate and obtain the exponential attractor in $L^{p}(\Omega) \times L^{q}(\Gamma)$. Finally, we show the exponential attractor in $\left(H^{1}(\Omega) \cap L^{p}(\Omega)\right) \times L^{q}(\Gamma)$.

\section{Introduction}

Parabolic equations with dynamical boundary conditions have strong backgrounds in mathematical physics. They arise in the heat transfer theory in a solid in contact with moving fluid, thermoelasticity, diffusion phenomena, heat transfer in two medium, problems in fluid dynamic, and so forth. At present, there are many monographs in the whole world (see [1-13]). Several approaches have been used for these equations, like the theory of semigroup, with Bessel potential and Besov space, and the variational setting. In particular, we are devoted to the long-time behavior of the solutions. For instance, In [1], the authors showed existence of pullback attractors. In $[8,9]$, the authors gave well posedness and global attractors in $L^{p}(\Omega) \times L^{p}(\Gamma)$. In [12, 13]; the authors obtained uniform attractors and some asymptotic regularity of global attractors in $\left(H^{1}(\Omega) \cap L^{p}(\Omega)\right) \times L^{q}(\Gamma)$. An exponential attractor, in contrast to a global attractor (or a uniform attractor), enjoys a uniform exponential rate of convergence of its solution. Because of this, exponential attractors possess more practical property. But to our knowledge, it does not seem to be in the literature any study of the existence of exponential attractors for this kind of equations.

This paper is concerned with existence of exponential attractors for the following reaction-diffusion equation with dynamic boundary condition

$$
\begin{gathered}
u_{t}-\Delta u+f(u)=h(x) \quad \text { in } \Omega, \\
u_{t}+\frac{\partial u}{\partial v}+g(u)=\rho(x) \quad \text { on } \Gamma, \\
u(0, x)=u_{0}(x), \quad \text { in } \bar{\Omega},
\end{gathered}
$$

where $\Omega \subset \mathbb{R}^{N}, N \geq 1$, is a bounded domain with a smooth boundary $\Gamma$. Here $v$ is the outer unit normal on $\Gamma$.

In [14], the authors established some necessary and sufficient conditions for the existence of exponential attractors for continuous and norm-to-weak continuous semigroup and provided a new method for proving the existence of exponential attractors by combining with the flattering property. Motivated by some ideas in [14-16], we combine asymptotic a prior estimate with the enhanced flattening property and show sufficient and necessary existence of exponential attractors in uniformly convex Banach spaces. As an application, we prove the existence of exponential attractors for the reaction-diffusion equation with dynamic boundary condition.

This paper is organized as follows. In Section 2, we recall some basic results and then give our theorems, that is, Theorems 5, 6, and 9 and the solution semigroup corresponding to (1). In Section 3, we obtain the exponential 
attractor in $L^{2}(\Omega) \times L^{2}(\Gamma)$ for weak solutions, then combine asymptotical a prior estimate and show the exponential attractor in $L^{p}(\Omega) \times L^{q}(\Gamma)$. Finally, we derive the existence of the exponential attractor in the space $\left(H^{1}(\Omega) \cap L^{p}(\Omega)\right) \times$ $L^{q}(\Gamma)$.

In the following, the constants $C_{i}, i=0,1,2, \ldots$ will always denote generic constants different in various occurrences. The symbol $C(s)$ will denote a positive constant dependent of $s$. We will write $\Omega_{T}$ for $\Omega \times(0, T), \Gamma_{T}$ for $\Gamma \times(0, T),(\cdot, \cdot)$ for the inner product in $L^{2}(\Omega)$ and $\langle\cdot, \cdot\rangle$ for the inner product in $L^{2}(\Gamma)$. For convenience, we denote the norm of $u$ by $|u|_{\Omega}$ in the space $L^{2}(\Omega)$ and $|u|_{\Gamma}$ in the space $L^{2}(\Gamma)$.

\section{Preliminary}

2.1. The Basic Results and Theorems. Let $X$ be a complete metric space and a one-parameter family of mappings $S(t)$ : let $X \rightarrow X(t \geq 0)$ be a semigroup. Here we omit the definitions of continuous or norm-to-weak semigroups, dynamical systems, global attractors, and exponential attractors (see [1520]).

Definition 1. Let $X$ be a metric space and $B$ be a bounded subset of $X$. The Kuratowski measure of noncompactness $\alpha(B)$ of $B$ is defined as

$$
\begin{gathered}
\alpha(B)=\inf \{\delta>0 \mid B \text { admits a finite cover by sets } \\
\text { of diameter } \leq \delta\} .
\end{gathered}
$$

Theorem 2 (see [14]). Assume that B is a bounded absorbing set for discrete dynamical system $S(n)$ in $X$; then the following are equivalent.

(1) The measure of noncompactness is exponentially decaying for dynamical system $(S(n), X)$, that is, there exist $k, l>0$ such that $\alpha\left(\bigcup_{m>n} S(m) B\right) \leq k e^{-l n}$.

(2) For $S(n)$, there exist exponential attractors.

Theorem 3 (see [14]). Assume that $B$ is a bounded absorbing set for $S(t)$ in $X$; then the following are equivalent.

(1) The measure of noncompactness is exponentially decaying for dynamical system $(S(t), X)$, that is, there exist $k, l>0$ such that $\alpha\left(\bigcup_{s>t} S(s) B\right) \leq k e^{-l t}$.

(2) For $S(t)$, there exist exponential attractors.

Definition 4 (see [14] (Enhanced Flattening Property)). Let $X$ be a uniformly convex Banach space; for any bounded set $B$ of $X$, there exist $k, l>0$ and $T>0$, and a finite dimension subspace $X_{1}$ of $X$, such that

(1) $P_{m}\left(\bigcup_{s \geq t} S(s) B\right)$ is bounded and

(2) $\left\|\left(I-P_{m}\right)\left(\bigcup_{s \geq t} S(s) x\right)\right\| \leq k e^{-l t}+k(m)$, for all $x \in B$, for all $t \geq T$.Here $P_{m}: X \rightarrow X_{1}$ is a bounded projector, $m$ is the dimension of $X_{1},\|\cdot\|$ denotes the norm in $X$, and $k(s)$ is a real-valued function satisfying $\lim _{s \rightarrow \infty} k(s)=0$.

Inspired by $[14,21]$, we easily obtain the following.

Theorem 5. Let $X$ be a uniformly convex Banach space and $\{S(t)\}_{t \geq 0}$ be a continuous or norm-to-weak continuous semigroup in $X$. Then the following conditions are equivalent.

(1) The measure of noncompactness is exponentially decaying for dynamical system $(S(t), X)$, that is, there exist $k, l>0$ such that $\alpha\left(\bigcup_{s>t} S(s) B\right) \leq k e^{-l t}$.

(2) $S(t)$ satisfies the enhanced flattening property.

Proof. ( $1 \Rightarrow 2$ ) On account of condition (1), for any bounded subset $B$ of $X$ and for any $\varepsilon>0$, there exist $t=t(B)$ such that

$$
\alpha\left(\bigcup_{s>t} S(s) B\right) \leq k e^{-l t} .
$$

Namely, there exist a finite number of subset $A_{1}, A_{2}, \ldots, A_{n}$ with diameter less than $k e^{-l t}$, such that

$$
\bigcup_{s>t} S(s) B \subset \cup_{i=1}^{n} A_{i}
$$

Let $x_{i} \in A_{i}$, then

$$
\bigcup_{s>t} S(s) B \subset \cup_{i=1}^{n} N\left(x_{i}, k e^{-l t}\right) \text {. }
$$

Let $X_{1}=\operatorname{span}\left\{x_{1}, x_{2}, \ldots, x_{n}\right\}$, since $X$ is uniformly convex, there exist a projection $P: X \rightarrow X_{1}$, such that for any $x \in X$, $\|x-P x\|=\operatorname{dist}\left(x, X_{1}\right)$. Hence,

$$
\|(I-P) S(t) x\| \leq k e^{-l t}<k e^{-l t}+k(m) .
$$

$(2 \Rightarrow 1)$ have been shown in Theorem 4.3 of [14], so we omitted it here.

By Theorem 5, we can deduce the following.

Theorem 6. Let $X$ be a uniformly convex Banach space and $\{S(t)\}_{t \geq 0}$ be a continuous or norm-to-weak continuous semigroup in $X$. Then, for dynamical system $(S(t), X)$, there exist exponential attractors in $X$ if and only if

(1) there is a bounded absorbing set $B \subset X$, and

(2) $S(t)$ satisfies the enhanced flattening property.

In addition, we use later the following theorem about global attractors.

Theorem 7 (see [15, Corollary 5.7]). Let $\{S(t)\}_{t \geq 0}$ be a semigroup on $L^{p}(\Omega),(p \geq 1)$, be a continuous or weak continuous semigroup on $L^{q}(\Omega)$ for some $q \leq p$, and have a global attractor in $L^{q}(\Omega)$. Then, $\{S(t)\}_{t \geq 0}$ has a global attractor in $L^{p}(\Omega)$ if and only if 
(1) $\{S(t)\}_{t \geq 0}$ has a bounded absorbing set $B_{0}$ in $L^{p}(\Omega)$, and

(2) for any $\varepsilon>0$ and any bounded subset $B \subset L^{p}(\Omega)$, there exist positive constants $M=M(, B)$ and $T=T(, B)$, such that

$$
\int_{\Omega\left(\left|S(t) u_{0}\right| \geq M\right)}\left|S(t) u_{0}\right|^{p}<\varepsilon \quad \text { for any } u_{0} \in B, t \geq T
$$

where $\Omega(|u| \geq M)=\{x \in \Omega|| u(x) \mid \geq M\}$.

We give the following lemma concerning the covering of the set in two different topologies used later in the proof of Theorem 9.

Lemma 8 (see [15, Lemma 5.3]). For any $\varepsilon>0$, the bounded subset $B$ of $L^{p}(\Omega)$ has a finite $\varepsilon$-net in $L^{p}(\Omega)$ if there exists a positive constant $M=M(\varepsilon)$, such that

(1) B has a finite $(3 M)^{(q-p) / q}(\varepsilon / 2)^{p / q}$-net in $L^{q}(\Omega)$;

(2) $\int_{\Omega(|u| \geq M)}|u|^{p} \leq 2^{-2 p+2} \varepsilon$, for any $u_{0} \in B_{0}$.

Inspired by [16], we give the subsequent theorem which describes our new technique to construct an exponential attractor in a stronger topological space.

Theorem 9. Assume that $p>q>0$ and $\Omega \subset \mathbb{R}^{n}$. Let $S(t)$ be a continuous or norm-to-weak continuous semigroup on $L^{p}(\Omega)$ and $L^{q}(\Omega)$ and $B_{0}$ be a positively invariant bounded absorbing set in $L^{p}(\Omega)$. If the following conditions hold true:

(1) $S(t)$ has an exponential attractor in $L^{q}(\Omega)$;

(2) for any $\varepsilon>0$, there exist positive constant $M=M(\varepsilon)$ such that

$$
\int_{\Omega\left(\left|S(t) u_{0}\right| \geq M\right)}\left|S(t) u_{0}\right|^{p} \leq 2^{-2 p+2} \varepsilon, \quad \text { for any } u_{0} \in B_{0} \text {, }
$$

then $S(t)$ has an exponential attractor in $L^{p}(\Omega)$.

Proof. Take $T>0$, and let $S^{n}=S(n T)$; obviously $S^{n}$ is a discrete dynamical system. On account of Condition (1), for $S^{n}$ there exists an exponential attractor $\mathscr{M}$ in $L^{q}(\Omega)$. By Theorem 2, we find that there exist $k, l>0$ such that $\alpha\left(\bigcup_{m>n} S(m) B\right) \leq k e^{-l n}$. By the definition of the measure of noncompactness, for all $n \in N$, there exist finite points $x_{n_{i}} \in S(n) B$ such that $S(n) B \subset \cup_{i=1}^{M_{n}}\left(B_{L^{q}(\Omega)}\left(x_{n_{i}}, k e^{-l n}\right) \cap S(n) B\right)$. Then, there exist $n_{0} \in \mathbb{N}$ and $M_{1}=C \varepsilon^{\gamma /(q-\gamma)}(\gamma>q)$ such that

$$
k e^{-l n_{0}} \leq\left(3 M_{1}\right)^{(q-p) / q}\left(\frac{\varepsilon}{2}\right)^{p / q} \leq k e^{-l\left(n_{0}-1\right)} .
$$

From Lemma 8 and Condition (2), it follows that $S\left(n_{0}\right)(B)$ has a finite $\varepsilon$-net in $L^{p}(\Omega)$.

Let $\varepsilon$ be replaced by $\varepsilon / 2$, there $n_{1} \in \mathbb{N}$ and $M_{2}=$ $C(\varepsilon / 2)^{\gamma /(q-\gamma)}(\gamma>q)$ such that

$$
k e^{-l\left(n_{0}+n_{1}\right)} \leq\left(3 M_{2}\right)^{(q-p) / q}\left(\frac{\varepsilon}{2^{2}}\right)^{p / q} \leq k e^{-l\left(n_{0}+n_{1}-1\right)} .
$$

From Lemma 8 and Condition (2), it follows that $S\left(n_{0}+n_{1}\right)(B)$ has a finite $\varepsilon / 2$-net in $L^{p}(\Omega)$.

By induction, let $\varepsilon / 2^{i-1}$ be replaced by $\varepsilon / 2^{i}$, there $n_{i} \in \mathbb{N}$ and $M_{i+1}=C\left(\varepsilon / 2^{i}\right)^{\gamma /(q-\gamma)}(\gamma>q)$ such that

$$
\begin{aligned}
k e^{-l\left(n_{0}+n_{1}+\cdots+n_{i}\right)} & \leq\left(3 M_{i+1}\right)^{(q-p) / q}\left(\frac{\varepsilon}{2^{i+1}}\right)^{p / q} \\
& \leq k e^{-l\left(n_{0}+n_{1}+\cdots+n_{i}-1\right)} .
\end{aligned}
$$

From Lemma 8 and Condition (2), it follows that $S\left(n_{0}+n_{1}+\right.$ $\left.\cdots+n_{i}\right)(B)$ has a finite $\varepsilon / 2^{i}$-net in $L^{p}(\Omega)$. Note that

$$
\begin{gathered}
\frac{\left(3 M_{i+1}\right)^{(q-p) / q}\left(\varepsilon / 2^{i+1}\right)^{p / q}}{\left(3 M_{i}\right)^{(q-p) / q}\left(\varepsilon / 2^{i}\right)^{p / q}} \\
=\frac{\left(M_{i+1} / M_{i}\right)^{(q-p) / q}}{2^{p / q}} \\
=\frac{\left(\left(\varepsilon / 2^{i}\right)^{\gamma /(q-\gamma)} /\left(\varepsilon / 2^{i-1}\right)^{\gamma /(q-\gamma)}\right)^{(q-p) / q}}{2^{p / q}} \\
=\left(\frac{1}{2}\right)^{\gamma(p-q) / q(\gamma-q)+p / q}<1 .
\end{gathered}
$$

So, the ratio of $\left(3 M_{i+1}\right)^{(q-p) / q}\left(\varepsilon / 2^{i+1}\right)^{p / q}$ and $\left(3 M_{i}\right)^{(q-p) / q}\left(\varepsilon / 2^{i}\right)^{p / q}$ is a constant for given $\gamma, p, q$, and $\varepsilon$. In fact,

$$
\begin{aligned}
\left(3 M_{i+1}\right)^{(q-p) / q}\left(\frac{\varepsilon}{2^{i+1}}\right)^{p / q} \\
=\left(\frac{1}{2}\right)^{p / q}(3 C)^{(q-p) / q} \varepsilon^{(\gamma(p-q) / q(\gamma-q))+p / q} \\
\quad \times\left(\frac{1}{2}\right)^{((\gamma(p-q) / q(\gamma-q))+p / q) i} .
\end{aligned}
$$

Substituting (13) into (11), we deduce easily that

$$
\begin{aligned}
\frac{\varepsilon}{2^{i}} \leq & 2\left(3 M_{i+1}\right)^{(p-q) / q} k^{q / p} e^{-q l\left(n_{0}+n_{1}+\cdots+n_{i}-1\right) / p} \\
= & 2(3 C)^{(q-p) / q} \varepsilon^{\gamma(p-q) / q(\gamma-q)}\left(\frac{1}{2}\right)^{(\gamma(p-q) / q(\gamma-q)) i} \\
& \times k^{q / p} e^{-q l\left(n_{0}+n_{1}+\cdots+n_{i}-1\right) / p} .
\end{aligned}
$$

Choose that $2(3 C)^{(q-p) / q} \varepsilon^{\gamma(p-q) / q(\gamma-q)} k^{q / p} e^{q l / p}=k^{\prime} \geq$ $2(3 C)^{(q-p) / q} \varepsilon^{\gamma(p-q) / q(\gamma-q)}(1 / 2)^{(\gamma(p-q) / q(\gamma-q)) i} k^{q / p} e^{q l / p}$ and $l^{\prime}=$ $q l / p$, we know that $S\left(n_{0}+n_{1}+\cdots+n_{i}\right)(B)$ has a finite $k^{\prime} e^{-l^{\prime}\left(n_{0}+n_{1}+\cdots+n_{i}\right)}$-net in $L^{p}(\Omega)$.

Combining (9), (10) (11), and (13), we can choose $n_{1}=$ $n_{2}=\cdots=n_{i}=[(1 / l)(\gamma(p-q) / q(\gamma-q)+p / q) \ln 2]+1$, where $[a]$ is the integer part of $a$. Denoted by $l_{0}=[(1 / l)(\gamma(p-$ $q) / q(\gamma-q)+p / q) \ln 2]+1$, we have $S\left(n_{0}+n_{1}+\cdots+\right.$ $\left.n_{i}\right)(B)=S\left(n_{0}+i l_{0}\right)$ has a finite $k^{\prime} e^{-l^{\prime}\left(n_{0}+i l_{0}\right)}$-net in $L^{p}(\Omega)$. By Theorem 2 , an exponential attractor exists in $L^{p}(\Omega)$ for discrete semigroup $S\left(i l_{0}\right)$. Using the same argument as in [18], 
it is easy to deduce that an exponential attractor $\mathscr{M}$ exists in $L^{p}(\Omega)$ for discrete semigroup $S(n)$. Let $\mathscr{M}_{0}=\cup_{0 \leq s \leq T} S(s) \mathscr{M}$. As an repetition of the general method developed by Li et al. [14], we can show that $\mathscr{M}_{0}$ is the exponential attractor for dynamical system $(S(t), X)$.

2.2. The Solution Semigroup. We can write Problem (1) as an evolution for unknown $u(x, t)$ in $\Omega$ and $v(x, t)$ on $\Gamma$

$$
w_{t}+A w+F=0,
$$

with the compatibility $v=\gamma(u(t))$ ( $\gamma$ is trace operator) for $t>0$ and $\lambda>0$, where

$$
\begin{gathered}
w=\left(\begin{array}{l}
u \\
v
\end{array}\right), \quad A=\left(\begin{array}{cc}
-\Delta+\lambda & 0 \\
\frac{\partial}{\partial \nu} & 0
\end{array}\right), \\
F=\left(\begin{array}{c}
f(u)-\lambda u-h(x) \\
g(v)-\rho(x)
\end{array}\right) .
\end{gathered}
$$

In the case $\Omega$ bounded, the operator $A$ has a compact resolvent and its spectrum, denoted by $\sigma(A)=\left\{\mu_{n}\right\}_{n} \subset R^{+}$, forms an increasing sequence converging to infinity (see [11, Theorem 1.4]). Moreover, there exists an orthonormal basis in $L^{2}(\Omega) \times L^{2}(\Gamma),\left\{\omega_{n}\right\}_{n}$, which are solutions of the eigenvalue problem

$$
\begin{gathered}
-\Delta u+\lambda u=\mu_{n} u, \quad x \in \Omega, \\
\frac{\partial u}{\partial v}=\mu_{n} u, \quad x \in \Gamma,
\end{gathered}
$$

where $\mu_{n} \subset R^{+}$forms an increasing sequence converging to infinity. We denote by $P_{n}$ the orthonormal projector

$$
\begin{gathered}
P_{n}: L^{2}(\Omega) \times L^{2}(\Gamma) \longrightarrow \operatorname{span}\left\{\omega_{1}, \omega_{2}, \ldots, \omega_{n}\right\}, \\
u=\sum_{i=1}^{\infty} \alpha_{i} \omega_{i} \longmapsto u_{n}=\sum_{i=1}^{n} \alpha_{i} \omega_{i} .
\end{gathered}
$$

So, we can perform the Galerkin truncation by using orthonormal basis mentioned above and guarantee the following existence and uniqueness (see $[1,8,9,12])$.

Theorem 10. Assume that the functions $f, g \in \mathscr{C}^{1}$ satisfying

$$
\begin{gathered}
-C_{0}+C_{1}|s|^{p} \leq f(s) s \leq C_{0}+C_{2}|s|^{p}, \quad p \geq 2, \\
-C_{3}+C_{4}|s|^{q} \leq g(s) s \leq C_{3}+C_{5}|s|^{q}, \quad q \geq 2, \\
f^{\prime}(s) \geq-l, \quad g^{\prime}(s) \geq-m .
\end{gathered}
$$

Then, Problem (1) has a unique weak solution, for any $T>0$, given $\left(u_{0}, \gamma\left(u_{0}\right)\right) \in L^{2}(\Omega) \times L^{2}(\Gamma)$ and $h(x) \in L^{2}(\Omega), \rho(x) \epsilon$ $L^{2}(\Gamma)$ there exists a solution $u$ with

$$
\begin{gathered}
u \in \mathscr{C}\left([0, T] ; L^{2}(\Omega)\right), \\
u \in L^{2}\left(0, T ; H^{1}(\Omega)\right) \cap L^{p}\left(\Omega_{T}\right), \\
u \in \mathscr{C}\left([0, T] ; L^{2}(\Gamma)\right), \quad u \in L^{q}\left(\Gamma_{T}\right), \\
\text { and }\left(u_{0}, \gamma\left(u_{0}\right)\right) \mapsto(u(t), \gamma(u)) \text { is continuous on } L^{2}(\Omega) \times L^{2}(\Gamma) .
\end{gathered}
$$

By the last theorem, we can define the operator semigroup $\{S(t)\}_{t \geq 0}$ in $L^{2}(\Omega) \times L^{2}(\Gamma)$ as follows:

$$
S(t)\left(u_{0}, \gamma\left(u_{0}\right)\right): L^{2}(\Omega) \times L^{2}(\Gamma) \times \mathbb{R}^{+} \longrightarrow L^{2}(\Omega) \times L^{2}(\Gamma),
$$

which is continuous in $L^{2}(\Omega) \times L^{2}(\Gamma)$.

Furthermore, we obtained the bounded absorbing set for dynamical system $(S(t), X)$ (see $[8,12])$.

Theorem 11. Under the assumptions of Theorem 10, the semigroup $\{S(t)\}_{t \geq 0}$ have $\left(L^{2}(\Omega) \times L^{2}(\Gamma), L^{p}(\Omega) \times L^{q}(\Gamma)\right)$-, $\left(L^{2}(\Omega) \times\right.$ $\left.L^{2}(\Gamma), H^{1}(\Omega) \times L^{q}(\Gamma)\right)$-bounded absorbing sets, that is, for any bounded subset $B \subset L^{2}(\Omega) \times L^{2}(\Gamma)$, there exists a positive constant $T$, which is only dependent on the $L^{2}(\Omega) \times L^{2}(\Gamma)$-norm of $B$, such that

$$
\begin{gathered}
\|u(t)\|_{L^{p}(\Omega)} \leq R_{0} \quad \text { for any }\left(u_{0}, \gamma\left(u_{0}\right)\right) \in B, t \geq T, \\
\|u(t)\|_{L^{q}(\Gamma)} \leq R_{0} \quad \text { for any }\left(u_{0}, \gamma\left(u_{0}\right)\right) \in B, t \geq T, \\
\|\nabla u(t)\|_{L^{2}(\Omega)} \leq R_{0} \quad \text { for any }\left(u_{0}, \gamma\left(u_{0}\right)\right) \in B, t \geq T,
\end{gathered}
$$

where $R_{0}$ is a positive constant independent of $B, u(t)=S(t) u_{0}$.

\section{The Main Results}

\subsection{Exponential Attractors in $L^{2}(\Omega) \times L^{2}(\Gamma)$}

Theorem 12. Under the hypothesis of Theorem 11, $\{S(t)\}_{t \geq 0}$ has $a\left(L^{2}(\Omega) \times L^{2}(\Gamma), L^{2}(\Omega) \times L^{2}(\Gamma)\right)$-exponential attractor.

Proof. Let $u_{2}=\left(I-P_{n}\right) u$, where $P_{n}$ is denoted by the orthonormal projector as mentioned before. Multiplying (1) by $u_{2}$ and integrating by parts, we get

$$
\begin{aligned}
\frac{1}{2} \frac{d}{d t}( & \left.\left\|u_{2}\right\|_{\Omega}^{2}+\left\|u_{2}\right\|_{\Gamma}^{2}\right) \\
& +\int_{\Omega}\left|\nabla u_{2}\right|^{2}+\int_{\Omega} f(u) u_{2}+\int_{\Gamma} g(u) u_{2} \\
= & \left(h, u_{2}\right)+\left\langle\rho, u_{2}\right\rangle .
\end{aligned}
$$

Using (19), we can deduce that

$$
|u|^{p} \geq|u|^{2}-C, \quad|u|^{q} \geq|u|^{2}-C,
$$

for any $p, q \geq 2$. Therefore,

$$
\begin{aligned}
\int_{\Omega} f(u) u_{2}+\int_{\Gamma} g(u) u_{2} \\
\quad \geq \int_{\Omega}\left|u_{2}\right|^{p}+\int_{\Gamma}\left|u_{2}\right|^{q}-C(m(\Omega), m(\Gamma)) .
\end{aligned}
$$

Thus, we know

$$
\begin{gathered}
\frac{d}{d t}\left(\left\|u_{2}\right\|_{\Omega}^{2}+\left\|u_{2}\right\|_{\Gamma}^{2}\right)+\mu_{n}\left(\int_{\Omega}\left|u_{2}\right|^{2}+\int_{\Gamma}|u|^{2}\right) \\
\leq C\left(m(\Omega), m(\Gamma),|h|_{\Omega},|\rho|_{\Gamma}\right) .
\end{gathered}
$$


Applying the Gronwall-inequality, we have

$$
\left\|u_{2}(t)\right\|_{\Omega}^{2}+\left\|u_{2}(t)\right\|_{\Gamma}^{2} \leq e^{-\mu_{n} t}+\frac{C}{\mu_{n}} .
$$

Obviously, the enhanced flattening property holds true. By Theorem 6, we obtain the exponential attractor in $L^{2}(\Omega) \times$ $L^{2}(\Gamma)$.

3.2. $\left(L^{2}(\Omega) \times L^{2}(\Gamma), L^{r}(\Omega) \times L^{r}(\Gamma)\right)$-Exponential Attractor. If $r=\min \{p, q\}$, we easily showed asymptotic a prior estimate of the solution of (1) in $L^{r}(\Omega) \times L^{r}(\Gamma)$ in order to obtain global and uniform attractors in $L^{p}(\Omega) \times L^{p}(\Gamma)$, where $r(\geq 2)$ is an integer (resp., see $[8,12]$ ).

Theorem 13. Under the hypothesis of Theorem 11, then for any $\varepsilon>0$ and any bounded subset $B \subset L^{2}(\Omega) \times L^{2}(\Gamma)$, there exist two positive constants $T=T(B, \varepsilon)$ and $M=M(\varepsilon)$ such that

$$
\begin{array}{r}
\int_{\Omega(|u| \geq M)}|u|^{r}+\int_{\Gamma(|u| \geq M)}|u|^{r} \leq C \varepsilon, \quad \forall t \geq T, \\
\left(u_{0}, \gamma\left(u_{0}\right)\right) \in B,
\end{array}
$$

where the constant $C$ is independent of $\varepsilon$ and $B$.

After Theorem 13, we obtain the subsequent result.

Theorem 14. Under the assumption of Theorem 11, then the semigroup $\{S(t)\}_{t \geq 0}$ generated by Problem (1) with initial data $u_{0} \in L^{2}(\Omega)$ and $\gamma\left(u_{0}\right) \in L^{2}(\Gamma)$ has a $\left(L^{2}(\Omega) \times L^{2}(\Gamma), L^{r}(\Omega) \times\right.$ $L^{r}(\Gamma)$ )-exponential attractor $\mathscr{M}_{r}$, that is, $\mathscr{M}_{r}$ is compact, invariant in $L^{r}(\Omega) \times L^{r}(\Gamma)$, and attracts every bounded in $L^{2}(\Omega) \times L^{2}(\Gamma)$ in the topology of $L^{r}(\Omega) \times L^{r}(\Gamma)$.

Proof. By Theorems 11 and 12 , it is easily verified that $\{S(t)\}_{t \geq 0}$ has an exponential attractor in $L^{r}(\Omega) \times L^{r}(\Gamma)$ where we apply Theorem 9.

If $r>\min \{p, q\}$, the reasoning process mentioned earlier is not available. We note the following result in [13] after authors obtained regularity of global attractor.

Lemma 15 (see [13, Corollary 3.1]). Under the assumptions of Theorem 11, the semigroup $\{S(t)\}_{t \geq 0}$ has a compact global attractor $\mathscr{A}$ in $\left(H^{1}(\Omega) \cap L^{p}(\Omega)\right) \times L^{q}(\Gamma)$. ing.

By Theorem 7 and Lemma 15, we easily obtain the follow-

Theorem 16. Under the hypothesis of Theorem 11, then for any $\varepsilon>0$ and any bounded subset $B \subset L^{2}(\Omega) \times L^{2}(\Gamma)$, there exist two positive constants $T=T(B, \varepsilon)$ and $M=M(\varepsilon)$ such that

$$
\begin{array}{r}
\int_{\Omega(|u| \geq M)}|u|^{p}+\int_{\Gamma(|u| \geq M)}|u|^{q} \leq C \varepsilon, \quad \forall t \geq T, \\
\left(u_{0}, \gamma\left(u_{0}\right)\right) \in B,
\end{array}
$$

where the constant $C$ is independent of $\varepsilon$ and $B$.
Applying Theorem 9 to Theorems 11 and 16, we have the following.

Corollary 17. Under the assumption of Theorem 12, the semigroup $\{S(t)\}_{t \geq 0}$ generated by Problem (1) with initial data $u_{0} \in$ $L^{2}(\Omega)$ and $\gamma\left(u_{0}\right) \in L^{2}(\Gamma)$ has a $\left(L^{2}(\Omega) \times L^{2}(\Gamma), L^{p}(\Omega) \times L^{q}(\Gamma)\right)$ exponential attractor $\mathscr{M}_{p q}$.

Remark 18. In fact, for any integer $r \leq \max \{p, q\}$, we can obtain Theorem 16 and Corollary 17 by Lemma 15 . In other words, if $r \leq \min \{p, q\}$, we can obtain Theorem 16 and Corollary 17 by Lemma 15 and need not use asymptotic a prior estimate. Here, we point this result obtained by the different procedure.

On account of Theorem 6 and Corollary 17, we have the following.

Corollary 19. There exists some $m$ such that

$$
\left\|\left(I-P_{m}\right) u\right\|_{L^{p}(\Omega)}+\left\|\left(I-P_{m}\right) u\right\|_{L^{q}(\Gamma)} \leq k e^{-l t}+k(m) .
$$

Now, we give some a prior estimates about $u_{t}$.

Lemma 20 (see $[8,12])$. Under the assumption of Theorem 11, for any bounded subset $B \subset L^{2}(\Omega) \times L^{2}(\Gamma)$, there exists a positive constant $T_{1}$ which depends only on the $L^{2}(\Omega) \times L^{2}(\Gamma)$ norm of $B$ such that

$$
\int_{\Omega}\left|u_{t}(s)\right|^{2} d x+\int_{\Gamma}\left|u_{t}(s)\right|^{2} d x \leq R_{1} \quad \forall s \geq T_{1}, \quad\left(u_{0,0}\right) \in B,
$$

where $R_{1}$ is a positive constant which depends on $M$.

We can easily obtain the following theorem.

Theorem 21. Under the assumption of Theorem 11, the semigroup $\{S(t)\}_{t \geq 0}$ generated by Problem (1) with initial data $u_{0} \in$ $L^{2}(\Omega)$ and $\gamma\left(u_{0}\right) \in L^{2}(\Gamma)$ satisfies the enhanced flattering in the space $H^{1}(\Omega) \times L^{q}(\Gamma)$.

Proof. We denote $u=u_{1}+u_{2}$, where $u_{1}=P_{n} u, u_{2}=\left(I-P_{n}\right) u$, and $P_{n}$ has been introduced in Section 3 .

Multiplying Problem (1) by $u_{2}$, we can get

$$
\begin{gathered}
\frac{1}{2} \frac{d}{d t} \int_{\Omega}\left|u_{2}\right|^{2}+\frac{1}{2} \frac{d}{d t} \int_{\Omega}\left|u_{2}\right|^{2}+\int_{\Omega}\left|\nabla u_{2}\right|^{2} \\
+\int_{\Omega} f(u) u_{2}+\int_{\Gamma} g(u) u_{2} \\
=\left(h, u_{2}\right)+\left\langle\rho, u_{2}\right\rangle .
\end{gathered}
$$


By (25) and (26), we can deduce

$$
\begin{aligned}
\int_{\Omega}\left|\nabla u_{2}\right|^{2} \leq & \left(\left|u_{t 2}\right|_{\Omega}+|h|_{\Omega}\right)\left|u_{2}\right|_{\Omega} \\
& +\left(\left|u_{t 2}\right|_{\Gamma}+|\rho|_{\Gamma}\right)\left|u_{2}\right|_{\Gamma} \\
& +\|u\|_{\Omega}^{p-1}\left\|u_{2}\right\|_{L^{p}(\Omega)} \\
& +\|u\|_{\Gamma}^{q-1}\left\|u_{2}\right\|_{L^{q}(\Gamma)} .
\end{aligned}
$$

By Theorems 11 and 12, Corollary 19, and Lemma 20, we know that $S(t) u_{0}$ satisfies the enhanced flattering property in $H^{1}(\Omega)$. On account of Theorem 6 and Corollary 19, we can deduce that Theorem 21 is valid.

It is immediate by Theorem 6, Corollary 19, and Theorem 21.

Corollary 22. Under the assumption of Theorem 12, the semigroup $\{S(t)\}_{t \geq 0}$ generated by Problem (1) with initial data $\left(u_{0}, \gamma\left(u_{0}\right)\right) \in L^{2}(\Omega) \times L^{2}(\Gamma)$ has a $\left(L^{2}(\Omega) \times L^{2}(\Gamma),\left(H^{1}(\Omega) \cap\right.\right.$ $\left.\left.L^{p}(\Omega)\right) \times L^{q}(\Gamma)\right)$-exponential attractor $\mathscr{M}$.

\section{Acknowledgment}

The research was supported by the NSFC Grant (no. 11271339).

\section{References}

[1] M. Anguiano, P. Marín-Rubio, and J. Real, "Pullback attractors for non-autonomous reaction-diffusion equations with dynamical boundary conditions," Journal of Mathematical Analysis and Applications, vol. 383, no. 2, pp. 608-618, 2011.

[2] J. M. Arrieta, P. Quittner, and A. Rodríguez-Bernal, "Parabolic problems with nonlinear dynamical boundary conditions and singular initial data," Differential and Integral Equations, vol. 14, no. 12, pp. 1487-1510, 2001.

[3] A. Rodríguez-Bernal, "Attractors for parabolic equations with nonlinear boundary conditions, critical exponents, and singular initial data," Journal of Differential Equations, vol. 181, no. 1, pp. 165-196, 2002.

[4] A. Constantin and J. Escher, "Global solutions for quasilinear parabolic problems," Journal of Evolution Equations, vol. 2, no. 1, pp. 97-111, 2002.

[5] A. Constantin and J. Escher, "Global existence for fully parabolic boundary value problems," Nonlinear Differential Equations and Applications, vol. 13, no. 1, pp. 91-118, 2006.

[6] A. Constantin, J. Escher, and Z. Yin, "Global solutions for quasilinear parabolic systems," Journal of Differential Equations, vol. 197, no. 1, pp. 73-84, 2004.

[7] J. Escher, "Quasilinear parabolic systems with dynamical boundary conditions," Communications in Partial Differential Equations, vol. 18, no. 7-8, pp. 1309-1364, 1993.

[8] Z. H. Fan and C. K. Zhong, "Attractors for parabolic equations with dynamic boundary conditions," Nonlinear Analysis: Theory, Methods \& Applications, vol. 68, no. 6, pp. 1723-1732, 2008.

[9] C. G. Gal and M. Warma, "Well posedness and the global attractor of some quasi-linear parabolic equations with nonlinear dynamic boundary conditions," Differential and Integral Equations, vol. 23, no. 3-4, pp. 327-358, 2010.
[10] J. Petersson, "A note on quenching for parabolic equations with dynamic boundary conditions," Nonlinear Analysis: Theory, Methods \& Applications, vol. 58, no. 3-4, pp. 417-423, 2004.

[11] L. Popescu and A. Rodriguez-Bernal, "On a singularly perturbed wave equation with dynamic boundary conditions," Proceedings of the Royal Society of Edinburgh A, vol. 134, no. 2, pp. 389-413, 2004.

[12] Y. Lu, "Uniform attractors for the closed process and applications to the reaction-diffusion equation with dynamical boundary condition," Nonlinear Analysis: Theory, Methods \& Applications, vol. 71, no. 9, pp. 4012-4025, 2009.

[13] L. Yang and M. Yang, "Long-time behavior of reaction-diffusion equations with dynamical boundary condition," Nonlinear Analysis: Theory, Methods \& Applications, vol. 74, no. 12, pp. 3876-3883, 2011.

[14] Y. Li, H. Wu, and T. Zhao, "Necessary and sufficient conditions for the existence of exponential attractors for semigroups, and applications," Nonlinear Analysis: Theory, Methods \& Applications, vol. 75, no. 17, pp. 6297-6305, 2012.

[15] C. K. Zhong, M. H. Yang, and C. Y. Sun, "The existence of global attractors for the norm-to-weak continuous semigroup and application to the nonlinear reaction-diffusion equations," Journal of Differential Equations, vol. 223, no. 2, pp. 367-399, 2006.

[16] Y. Zhong and C. Zhong, "Exponential attractors for reactiondiffusion equations with arbitrary polynomial growth," Nonlinear Analysis: Theory, Methods \& Applications, vol. 71, no. 3-4, pp. 751-765, 2009.

[17] L. Dung and B. Nicolaenko, "Exponential attractors in Banach spaces," Journal of Dynamics and Differential Equations, vol. 13, no. 4, pp. 791-806, 2001.

[18] A. Eden, C. Foias, B. Nicolaenko, and R. Temam, Exponential Attractors for Dissipative Evolution Equations, vol. 37 of Research in Applied Mathematics, John Wiley \& Sons, New York, NY, USA, 1994.

[19] M. Grasselli and D. Pražák, "Exponential attractors for a class of reaction-diffusion problems with time delays," Journal of Evolution Equations, vol. 7, no. 4, pp. 649-667, 2007.

[20] R. Temam, Infinite-Dimensional Dynamical Systems in Mechanics and Physics, vol. 68 of Applied Mathematical Sciences, Springer, New York, 1997.

[21] Q. Ma, S. Wang, and C. Zhong, "Necessary and sufficient conditions for the existence of global attractors for semigroups and applications," Indiana University Mathematics Journal, vol. 51, no. 6, pp. 1541-1559, 2002. 


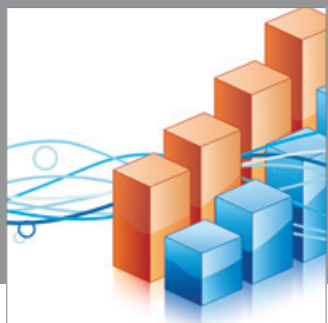

Advances in

Operations Research

mansans

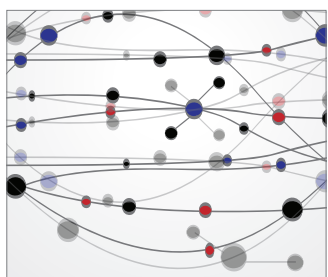

The Scientific World Journal
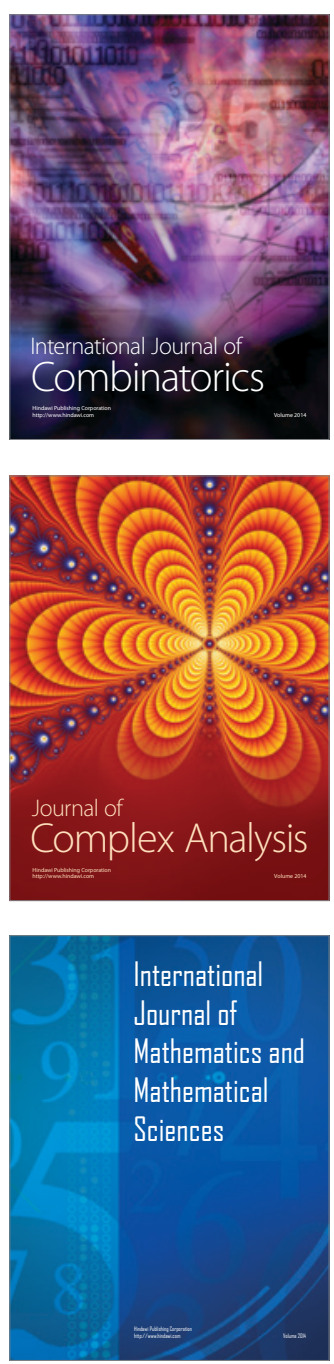
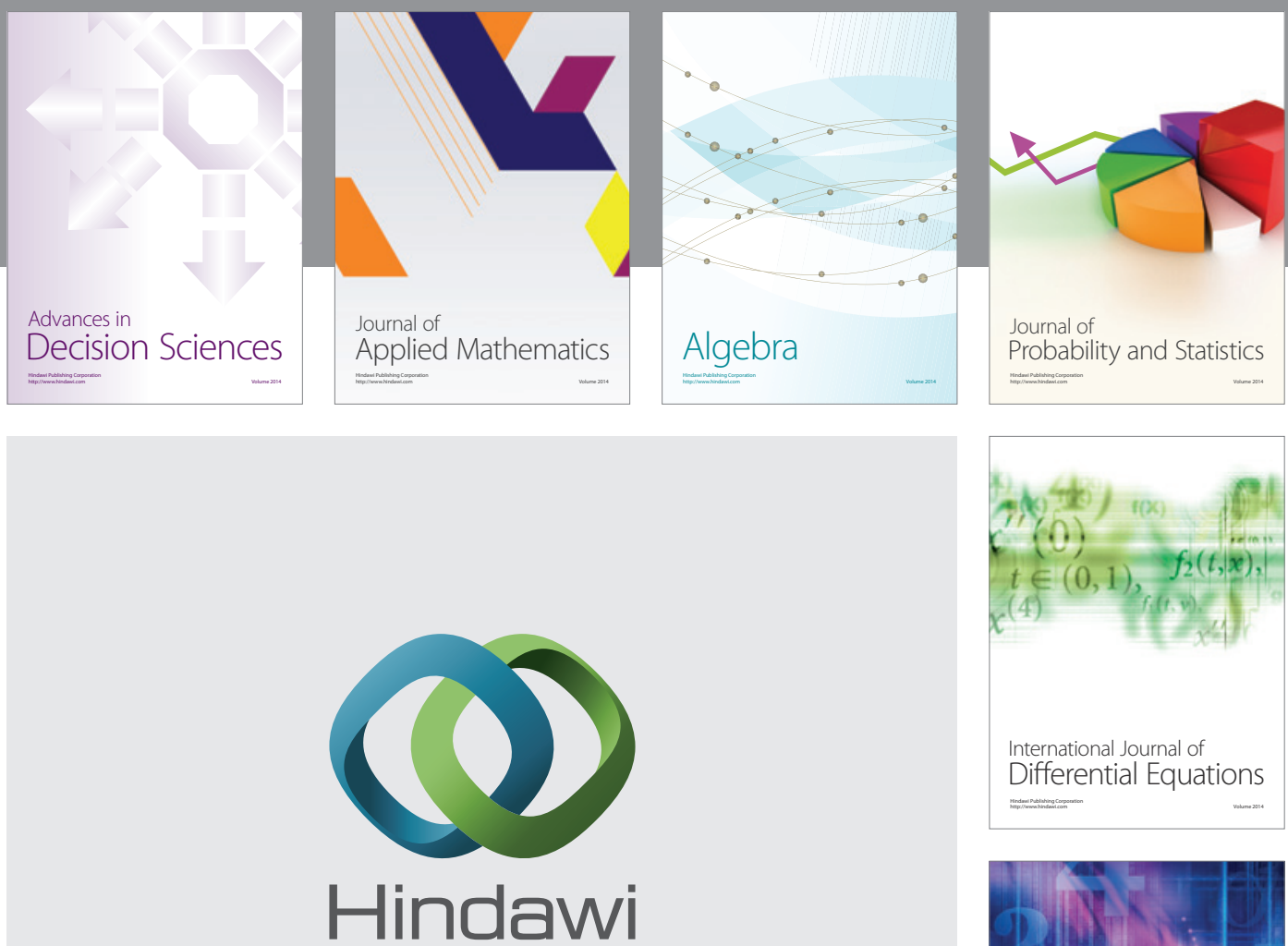

Submit your manuscripts at http://www.hindawi.com
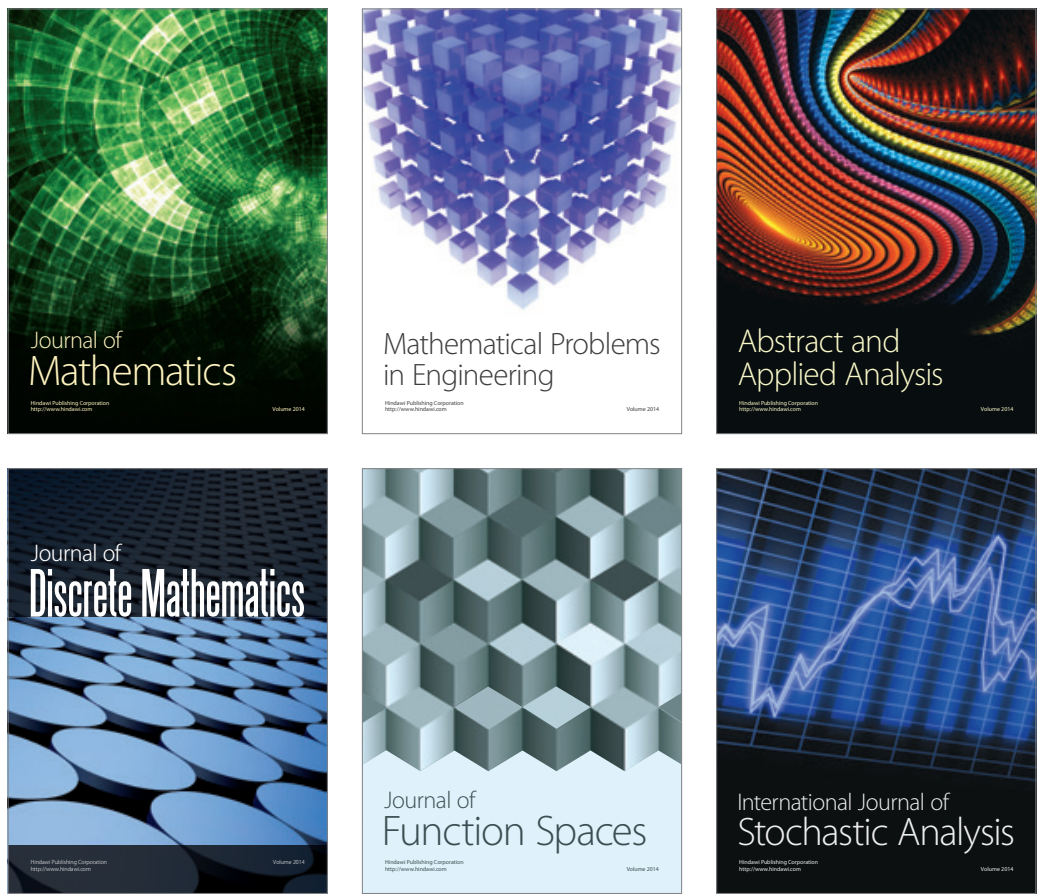

Journal of

Function Spaces

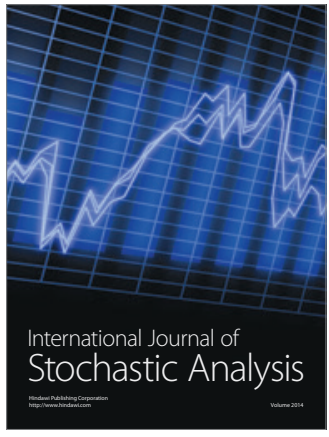

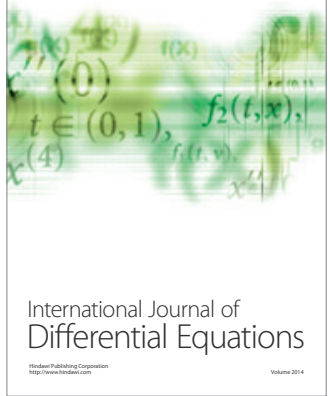
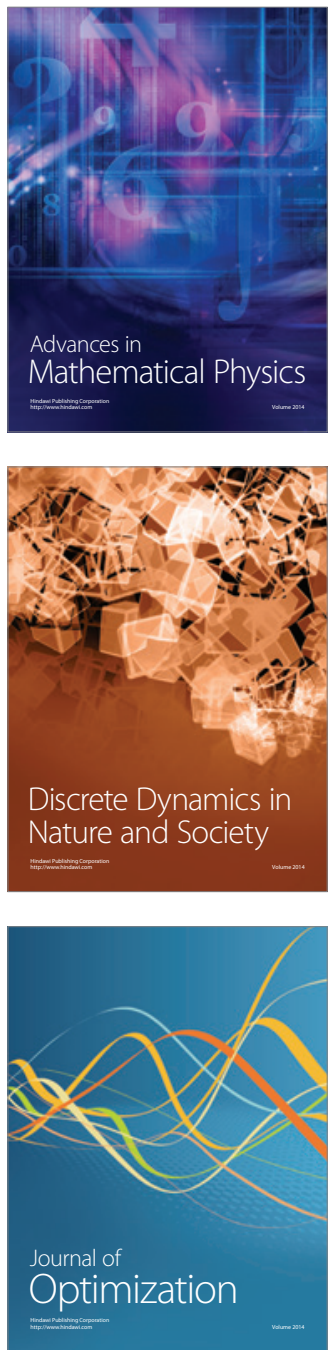